\title{
Compaired Sacubitril/Valsartan with Benalapril on the Left Ventricular Remodeling of Ami after PPCI: A case-control study
}

\author{
Yuan cao MD PhD ${ }^{1}$, Wei lian MD ${ }^{12}$, Pradeep Hathurusinghe $\mathrm{MD}^{1}$, DEEPARK SHARMA MD ${ }^{1}$ Yanmin XU MD PHD ${ }^{13^{*}}$ \\ ${ }^{1}$ Department of Cardiology, Tianjin Key Laboratory of Ionic-Molecular Function of Cardiovascular disease, PR China. \\ ${ }^{2}$ Tianjin Institute of Cardiology, Second Hospital of Tianjin Medical University, PR China. \\ ${ }^{3}$ No 23 Ping Jiang road of HE XI District Tianjin, PR China.
}

*Corresponding Author: Yanmin XU, Department of Cardiology, Tianjin Key Laboratory of Ionic-Molecular Function of Cardiovascular disease, Tianjin Institute of Cardiology, Second Hospital of Tianjin Medical University, No 23 Ping Jiang road of HE XI District Tianjin, PR China.

Received date: January 31 2021; Accepted date: February 19, 2021; Published date: March 01,2021

Citation: Yuan cao, Wei lian, Pradeep Hathurusinghe, DEEPARK SHARMA, Yanmin XU (2021) Compaired Sacubitril/Valsartan with Benalapril on the Left Ventricular Remordeling of Ami after PPCI: A case-control study. J. Clinical Research and Clinical Trials. 3(1); DOI: 10.31579/26934779/024

Copyright: (02021, Yanmin XU. This is an open access article distributed under the Creative Commons Attribution License, which permits unrestricted use, distribution, and reproduction in any medium, provided the original work is properly cited.

\begin{abstract}
:
Background: To compare the effect of sacubitril/valsartan with benalapril on left ventricular remodeling in patients with acute myocardial infarction.

Materials and Methods: 85 patients with acute ST segment elevation myocardial infarction who were treated with PCI in the Second Affiliated Hospital of Tianjin Medical University. The patients were randomly divided into two groups: the experimental group (sacubitril/valsartan, 25-100mg/d, BID) and the control group (benalapril, 5$10 \mathrm{mg}$, QD). Color Doppler echocardiography was performed after 1 month and 3 months respectively, interventricular septal thickness, septal motion amplitude, left ventricular end diastolic diameter, left ventricular end systolic diameter, posterior wall thickness, posterior wall motion amplitude, LVEF, left ventricular weight, left ventricular weight index, NT Pro-BNP, gender, height, weight, body surface area were collected. In order to evaluate the influencing factors in the process of ventricular remodeling, binary multivariate logistic regression analysis was carried out for the indicators with statistical differences in the conclusions of the above control study. Subgroup analysis was carried out and the samples were divided into four subgroups according to gender,age, initial ejection fraction .

Results: one month after the treatment of sacubitril/valsartan or benalapril, only the left ventricular end systolic diameter was statistically different between the two groups $(\mathrm{P}<0.05)$, and the other indexes were not statistically different. Three months after treatment with sacubitril/valsartan or benalapril, there were statistical differences in the indexes related to myocardial remodeling between the two groups $(\mathrm{P}<0.05)$. The results of multivariate logistic analysis showed that the index of left ventricular end systolic diameter was statistically significant (or=0.006, 95\% CI: 0.733-0.981). Acute myocardial infarction whose LVEF is less than 50\%, show sacubitril/valsartan is better than traditional ACEI.
\end{abstract}

Conclusion: sacubitril/valsartan Compared with benalapril is better on left ventricular remodeling in patients with ST segment elevation acute myocardial infarction.

Key words: sacubitril/valsartan; benalapril; left ventricular remordeling; AMI; PPCI

\section{Introduction}

Chronic heart failure is the end stage of many cardiovascular diseases. Development of the HF phenotype in these patients arise from a complex, progressive, molecular and cellular transformation references "ventricular remodeling", includes dilation of the ventricle, the formation of scar and geometrical charges in the overall left ventricle(LV) shape (i e, ellipsoid to more spherical) and is driven, in part by neurohormonal pathways. Left ventricular remodeling is characterized by a progressive increase in both end-diastolic (LVEDV) and end-systolic volumes (LVESV). The increase in LVESV can precede the increase in LVEDV, as a consequence of an impaired systolic function that causes a reduction in stroke volume. Post infarct remodeling is a specific type of left ventricular remodeling that is a consequence of an increase in both preload and afterload causing an enlargement of ventricular chamber and a hypertrophy of normal myocardium.

The over reaction of renin-angiotensin aldosterone system (RAAS) is an important driving factor. Anggiotensin II (Ang II) can promote the occurrence and development of ventricular remodeling in patients with cardiac insufficiency. As a compound preparation, sacubitrial/valsartan can inhibit the hydrolysis of brain natriuretic peptide and the biological 
activity of angiotensin-II, antagonize the over activation of neuroendocrine system, and finally achieve the effect of vasodilation, inhibition of ventricular remodeling and reduction of heart burden, The inhibition of brain natriuretic peptidase and the increase of natriuretic peptide level by sacubitril/valsartan (ARNI) is an important means to treat heart failure and fight against ventricular remodeling and the inhibition of RAAS system over activation is also of great significance to delay myocardial remodeling. The synergistic effect of the two mechanisms can significantly improve the therapeutic effect.

The purpose of this study is to explore the improvement of myocardial remodeling in patients with acute myocardial infarction after short-term treatment and long-term treatment by combining with traditional ACEI drugs.

\section{Subjects and methods}

\section{Research subjects}

85 patients with acute ST segment elevation myocardial infarction were enrolled who were treated with PCI in the Second Affiliated Hospital of Tianjin Medical University, the patients were randomly divided into two groups: the experimental group (ARNI 25mg-100mg; BID) and the protocol group (benalapril, 5-10mg; QD).

Inclusion/Exclusion criteria were according to the 2013 ACCF/AHA guideline for the management of ST-elexation myocardial infarction:executive summary: a report of the American college of cardiology foundation/American heart Association Task Force on Practice guideline.

\section{Study methods}

Gender,height, weight, body surface area, NT Pro- BNP were collected respectively, interventricular septal thickness, septal motion amplitude, left ventricular end diastolic diameter, left ventricular end systolic diameter, posterior wall thickness, posterior wall motion amplitude, LVEF, left ventricular weight, left ventricular weight index and were collected respectively using Color Doppler echocardiography after myocardial infarction 1 month and 3 months respectively.

\section{Clinical data analysis}

The clinical data of the subjects were collected and analyzed: first, the basic information of the patients, such as gender, height, weight, body surface area and other indicators were analyzed to ensure that there is no difference in the basic information of randomly enrolled patients. In addition, NT Pro-BNP, interventricular septal thickness, septal motion amplitude, left ventricular end diastolic diameter, left ventricular end systolic diameter, posterior wall thickness, posterior wall motion amplitude, LVEF, left ventricular weight, left ventricular weight index were analyzed. In order to evaluate the influencing factors in the process of ventricular remodeling, binary multivariate logistic regression analysis was carried out for the indicators with statistical differences in the conclusions of the above control study and the OR value was calculated. Sub group analysis was carried out and the samples were divided into three subgroups according to sex, age, initial ejection fraction. The intervention effect of ARNI on ventricular remodeling in different populations were studied.

\section{Statistical methods}

SPSS 21.0 software was used to analyze the clinical and examination data. Measurement data was expressed as mean \pm standard deviation and $\mathrm{T}$ test was used for comparison between groups. $\mathrm{P}<0.05$ was statistically significant. Logistic regression analysis was used to analyze the statistically significant parameter, $\mathrm{P}<0.05$ was statistically significant.

\section{Results}

85 patients with acute ST segment elevation myocardial infarction who were treated with PPCI in the Second Affiliated Hospital of Tianjin Medical University. The patients were randomly divided into two groups: the experimental group (sacubitril/valsartan), there were 34 males and 14 females and the control group (Benalapril). There were 27 males and 10 females. Average ages is $68.6 \pm 12.6$ years old.

After 1 month of treatment, there was a significant difference in the end systolic diameter between the two groups $(\mathrm{P}<0.05)$. There was no statistical significance in other clinical data, and there was no significant difference between the two treatments. Table 1

\begin{tabular}{|c|c|c|c|c|}
\hline Item & Experimental group & Control group & T & P \\
\hline NT-pro-BNP & $1560.23 \pm 1304.25$ & $1732.95 \pm 1612.93$ & 0.391 & 0.698 \\
\hline $\begin{array}{c}\text { Interventricular septal } \\
\text { thickness }\end{array}$ & $9.28 \pm 1.77$ & $9.61 \pm 2.27$ & 0.541 & 0.592 \\
\hline Range of septal motion & $7.16 \pm 3.17$ & $6.41 \pm 2.97$ & 0.816 & 0.419 \\
\hline $\begin{array}{c}\text { Left ventricular end } \\
\text { diastolic diameter }\end{array}$ & $52.97 \pm 6.76$ & $55.79 \pm 6.46$ & 1.411 & 0.166 \\
\hline $\begin{array}{c}\text { Left ventricular end } \\
\text { systolic diameter }\end{array}$ & $35.90 \pm 12.85$ & $45.50 \pm 8.55$ & 2.919 & 0.006 \\
\hline Back wall thickness & $9.21 \pm 1.69$ & $9.51 \pm 1.50$ & 0.623 & 0.536 \\
\hline $\begin{array}{c}\text { Back wall motion } \\
\text { mplitude }\end{array}$ & $8.31 \pm 3.26$ & $6.66 \pm 2.34$ & 1.929 & 0.061 \\
\hline Left ventricular weight & $49.18 \pm 14.00$ & $41.68 \pm 14.36$ & 1.754 & 0.087 \\
\hline $\begin{array}{c}\text { Left ventricular mass } \\
\text { index }\end{array}$ & $1890.48 \pm 674.66$ & $2031.47 \pm 536.22$ & 0.767 & 0.447 \\
\hline
\end{tabular}

Table 1: Comparison between Experimental group and control groups after 1 month of treatment.

Three months after treatment with sacubitril/valsartan or benalapril, there were statistical differences in the indexes related to myocardial remodeling between the two groups $(\mathrm{P}<0.05)$. Three months after treatment, the indexes of myocardial remodeling in the experimental group were better than those in the benalapril group. Table 2 


\begin{tabular}{|c|c|c|c|c|}
\hline Item & Experimental group & Control group & T & P \\
\hline NT-pro-BNP & $1044.44 \pm 938.18$ & $2672.73 \pm 525.73$ & 1.182 & 0.256 \\
\hline Interventricular septal thickness & $8.65 \pm 1.89$ & $9.53 \pm 1.53$ & 1.416 & 0.168 \\
\hline Range of septal motion & $8.58 \pm 2.58$ & $5.57 \pm 2.56$ & 3.195 & 0.003 \\
\hline Left ventricular end diastolic diameter & $50.19 \pm 2.80$ & $56.21 \pm 6.58$ & 3.351 & 0.002 \\
\hline Left ventricular end systolic diameter & $30.45 \pm 8.80$ & $44.85 \pm 9.53$ & 4.363 & 0.000 \\
\hline Back wall thickness & $9.08 \pm 1.36$ & $9.14 \pm 1.80$ & 0.112 & 0.912 \\
\hline Back wall motion amplitude & $9.96 \pm 1.80$ & $7.62 \pm 2.45$ & 3.000 & 0.006 \\
\hline Left ventricular weight & $54.81 \pm 59.90$ & $42.13 \pm 15.35$ & 2.713 & 0.012 \\
\hline Left ventricular mass index & $1690.02 \pm 435.88$ & $1912.55 \pm 550.69$ & 1.242 & 0.225 \\
\hline
\end{tabular}

Table 2: Comparison between Experimental group and control groups after 3 months of treatment

For acute myocardial infarction patients with LVEF $\leq 50 \%$, the treatment of ARNI is more effective than that of traditional drugs. For the patients with low left ventricular ejection fraction (LVEF $\leq 50 \%)$, most of them are anterior myocardial infarction, with large infarct area and serious myocardial damage. The cardiac function and structure were obviously abnormal. In the treatment, ARNI is better than traditional medicine. For the patients with higher left ventricular ejection fraction (LVEF $>50 \%$ ), most of them are inferior myocardial infarction. The left ventricular function and structure of the patients are not seriously damaged and there is no evidence of cardiac dysfunction. After the treatment of two drugs, there was no obvious abnormality in the indexes of myocardial remodeling in this group. Table 3

\begin{tabular}{|c|c|c|c|c|}
\hline Item & Experimental group & Control group & T & P \\
\hline NT-pro-BNP & $967.80 \pm 707.41$ & $3019.73 \pm 606.46$ & 0.740 & 0.472 \\
\hline Interventricular septal thickness & $8.19 \pm 1.91$ & $8.96 \pm 1.44$ & 0.798 & 0.438 \\
\hline Range of septal motion & $8.60 \pm 2.59$ & $4.67 \pm 2.02$ & 3.319 & 0.005 \\
\hline Left ventricular end diastolic diameter & $49.50 \pm 2.63$ & $58.25 \pm 5.38$ & 3.409 & 0.004 \\
\hline Left ventricular end systolic diameter & $35.62 \pm 2.29$ & $47.74 \pm 8.74$ & 3.001 & 0.010 \\
\hline Back wall thickness & $8.82 \pm 1.28$ & $8.97 \pm 1.47$ & 0.200 & 0.844 \\
\hline Back wall motion amplitude & $10.24 \pm 1.12$ & $7.14 \pm 2.54$ & 2.583 & 0.022 \\
\hline Left ventricular weight & $1547.74 \pm 403.07$ & $1962.06 \pm 606.74$ & 1.381 & 0.189 \\
\hline Left ventricular mass index & $926.46 \pm 190.97$ & $1197.90 \pm 457.34$ & 1.259 & 0.229 \\
\hline
\end{tabular}

Table 3: Comparison between Experimental group and control groups of patients with low left ventricular ejection fraction

The indexes with statistical differences, such as the amplitude of interventricular septal motion, the end diastolic diameter of left ventricle, the end systolic diameter of left ventricle, the amplitude of posterior wall motion and LVEF, were analyzed by logistic regression. The results of multivariate logistic analysis showed that the index of left ventricular end systolic diameter was statistically significant or $=0.006$ (95\% CI: 0.733 0.981 ), Left ventricular end diastolic diameter was dependently risk of remodeling. Table 4

\begin{tabular}{|l|l|l|l|l|}
\hline Item & B & X2 & P & OR(95\% CI) \\
\hline Range of motion of interval & -0.223 & 1.977 & 0.160 & $0.800(0.587-1.092)$ \\
\hline Left ventricular end diastolic diameter & 0.100 & 1.181 & 0.277 & $0.166(0.923-1.322)$ \\
\hline Left ventricular end systolic diameter & -0.165 & 4.909 & 0.027 & $0.006(0.733-0.981)$ \\
\hline Back wall motion amplitude & 0.161 & 1.342 & 0.247 & $0.061(0.895-1.542)$ \\
\hline LVEF & 0.004 & 0.014 & 0.905 & $0.087(0.942-1.070)$ \\
\hline
\end{tabular}

Table 4: multivariate logistic regression analysis

\section{Discussion}

Left ventricular remodeling is characterized by a progressive increase in both end-diastolic (LVEDV) and end-systolic volumes (LVESV). The increase in preload is sustained by the phenomenon of infarct expansion, which is an enlargement of infarct scar. This causes a segmental increase in the ventricular volume subtended by the expanded infarcted myocardial wall. In infarcted myocardium, ventricular contraction is not symmetrical, because the necrotic segments have lost their contractility. As a result, the force generated by the normal remote myocardium during contraction is not counterbalanced by an equal and opposite force, the infarcted ventricular wall is thus stretched by an increased wall tension that is not homogeneously distributed in the left ventricle [1]. Infarcted wall usually has longer contraction times than the healthy myocardium. This wall motion defect has been recognized as a risk factor for the development of remodeling, and it can be assessed with echocardiography or cine CMR to maintain a normal stoke volume with a reduced number of normally working myocardial segments, the healthy myocardium has to produce a greater pressure ${ }^{[2,3]}$. The increase in afterload on healthy myocardium causes a hypertrophy of cardiomyocytes. In post infarct ventricular remodeling, hypertrophic cardiomyocytes are longer than normal cardiac cells. Post infarct ventricular remodeling was characterized by a lengthening of cardiomyocytes especially in the area surrounding the infarct scar, but also in remote myocardium. This type of ventricular hypertrophy has been termed eccentric and contributes to the worsening of ventricular dilatation during remodeling $[4,5]$.Cardiac hypertrophy that occurs during post infarct remodeling is accompanied by an increase in extracellular matrix, which is mainly constituted by collagen. The results are due to an increased activity of cardiac fibroblasts in response to different soluble fibrogenic mediators, such as transforming growth factor-II (TGF-II) and systemic and local activation of renin-angiotensin aldosterone system (RAAS). The mediators of the RAAS that promote ventricular remodeling are angiotensin II and aldosterone. Remodeling is 
a pathologic process that involves the entire ventricle, leading to a change in its global structure. There are two types of causes of remodeling: mechanical and biochemical. While mechanical causes, as previously described, are an increase in both preload and afterload, biochemical causes are linked to the production of soluble mediators capable of promoting ventricular remodeling. antimyocardial remodeling therapy is very important for improving the prognosis of patients with myocardial infarction. The over reaction of RAAS system is an important driving factor. Angiotensin II can promote the occurrence and development of ventricular remodeling in patients with cardiac insufficiency [9]. Angiotensin II can not only promote cardiomyocyte hypertrophy and intercellular glial deposition, but also accelerate the process of cardiomyocyte apoptosis. Under the action of angiotensin II, c-myc, cjun, c-fos and other genes are highly expressed, which leads to the increase of fetal protein synthesis and cardiomyocyte proliferation. Angiotensin II can also promote the aggregation of inflammatory factors such as endothelin-1, tumor necrosis factor $\alpha$, interleukin (IL) and other related inflammatory factors under the guidance of at-1r [10]. Through cell signal transduction, angiotensin II can cause cardiac hypertrophy, apoptosis and myocardial fibrosis, and promote the process of ventricular remodeling.The increase of ventricular pressure can stimulate the production and release of natriuretic peptide, promote the excretion of water and sodium, reduce the myocardial pressure and slow down the process of myocardial remodeling ${ }^{[11]}$. ACEI and these effects play a key role in the process of myocardial remodeling. McMurray and others reports [12] showed that compared with the placebo group from the SOLVD-T study, the relative risk of cardiac end-point events in patients treated with ARNI was reduced by $43 \%$, of which the cardiovascular mortality rate was reduced by $34 \%$, the admission rate of heart failure was reduced by $49 \%$, and the all-cause mortality rate was reduced by $28 \%$.

At present, the related researches mostly use LVEDV, LVESV, LEDVI, LVEF, LVM, LVMI and other indicators to evaluate the degree of myocardial remodeling $[6,7,8]$. The indexes of LVEDV, LVESV and LVM in patients with acute myocardial infarction increased significantly, while LVEF and LVMI decreased significantly. Therefore, the experience of myocardial remodeling in patients with acute myocardial infarction can be roughly described as follows: firstly, the compliance of myocardial necrosis with loss of blood supply is decreased, and at the same time, it is affected by intracardiac pressure. At present, there are few related experiments on the treatment of acute myocardial infarction patients with sacubitril/valsartan instead of ACEI / ARB $[13,14,15]$. In this study, we compared the changes of echocardiography related indexes between ACEI and sacubitril/valsartan in patients with acute myocardial infarction after 1 month and 3 months. Sacubitril/valsartan has therapeutic effect on myocardial remodeling in patients with acute myocardial infarction and can improve the prognosis of cardiac function in such patients, but the treatment time is relatively short (1 month). The therapeutic effect is not significantly different from that of traditional ACEI and only has advantages on the improvement of left ventricular end systolic diameter. However, after a long time ( 3 months) treatment, the therapeutic effect of sacubitril/valsartan is better than that of ACEI in the treatment of myocardial remodeling in patients with acute myocardial infarction. There were significant improvement effects on the parameters such as interventricular septal motion amplitude, left ventricular end diastolic diameter, left ventricular end systolic diameter, posterior wall motion amplitude, LVEF. It may be related to the increase of brain natriuretic peptide level. In the subgroup analysis of this study, Most of the patients with acute myocardial infarction whose LVEF is less than $50 \%$ are anterior wall myocardial infarction, with large infarct area and serious myocardial damage. The cardiac function and structure were obviously abnormal. After treated with sacubitril/valsartan is better than traditional medicine. Most of the patients with LVEF greater than 50\% were inferior myocardial infarction. The left ventricular function and structure of the patients were not seriously damaged, and there was no evidence of cardiac insufficiency. After the treatment of two drugs, there was no obvious abnormality in the indexes of myocardial remodeling in two group.

Apoptosis of cardiomyocytes is an important pathophysiological mechanism of cardiac dysfunction in patients with acute myocardial infarction. The high expression of Fas and FasL can be measured in cardiomyocytes of rats with acute myocardial infarction, indicating that cardiomyocytes can also suffer from Fas 1 cytotoxic effect. The upregulation of Fas and FasL expression is the initiation and mediating process of myocardial necrosis and related inflammatory reaction after acute myocardial infarction. Myocardial necrosis and remodeling are common in patients with acute myocardial infarction. Myocardial remodeling will greatly affect the quality of life and prognosis of patients with myocardial infarction. Therefore, anti-myocardial remodeling therapy is very important for improving the prognosis of patients with myocardial infarction $[16,17,18]$.

As a compound preparation, sacubitril/valsartan can inhibit the hydrolysis of brain natriuretic peptide and the biological activity of angiotension-II, antagonize the over activation of neuroendocrine system, and finally achieve the effect of vasodilation, inhibition of ventricular remodeling and reduction of heart burden [19, 20].

There are still some shortcomings and many limitations exit in this study. First of all, the number of cases in this study is small, and the research results may be one-sided, which needs to be further studied by large samples and multi-centers. Secondly, most of the myocardial remodeling occurred in the patients with anterior myocardial infarction. In this study, the number of patients with anterior myocardial infarction was small, and the treatment effect of the patients with inferior myocardial infarction was negative in the comparative study, so there was a deviation in the evaluation of the therapeutic effect of sacubitril/valsartan. Finally, in this study, the dosage of sacubitril/valsartan did not fully comply with the guidelines and it was confirmed by experiments that the application of 4 weeks can have beneficial effects on myocardial remodeling and is linearly related to the dosage of the drug.

\section{References}

1. Ferrario CM, Schiffrin EL. (2015) Role of mineralocorticoid receptor antagonists in cardiovascular disease [J].116 (1): 206213.

2. Zannad F, Mcmurray JJ, Krum H, et al. (2011) Eplerenone in patients with systolic heart failure and mild symptoms [J]. N Engl J Med. 364 (1): 11-21.

3. Tendera M, Talajic M, Robertson M, et al. (2011) Safety of ivabradine in patient's withcoronary artery disease and left ventricular systolic dysfunction [J]. Am J Cardiol. 107 (6): 805811.

4. Kim HY, Bae EH, Ma SK, et al. (2014) Effects of spironolactone in combination with Angiotensin converting enzyme inhibitors or Angiotensin receptor blockers in patients with proteinuria [J]. Kidney Blood Press Res.39 (6): 573-580.

5. Pitt B, Zannad F, Remme WJ, et al. (1999) The effect of spironolactone on morbidity and mortality in patients with severe heart failure [J]. N Engl J Med. 341 (10): 709-717.

6. Swedberg K, Komajda M, Bhm M, et al. (2012) Effects on outcomes of heart rate reduction by ivabradine in patients with congestive heart failure: is there an influence of beta-blocker dose [J]. J Am Coll Cardiol. 59 (22) : 1938-1945.

7. Follath F, Cleland JG, Just H, et al. (2002) Efficacy and safety of intravenous levosimendan compared with dobutamine in severe 
low-output heart failure (the LIDO study): a randomised doubleblind trial [J]. Lancet.360 (9328) : 196-202.

8. Hees HW, Dekhuijzen PN, Heunks LM. (2009) Levosimendan enhances forcegeneration of diaphragm muscle from patients with chronic obstructive pulmonary disease [J]. Am J R espir Crit Care Med. 179 (1) : 41-47.

9. Shaffer JA, Thompson JL, Cheng B, et al. (2014) Association of quality of life with anticoagulant control in patients with heart failure: the Warfarin and Aspirin in reduced Cardiac Ejection Fraction (WARCEF) trial [J]. Int J Cardiol. 177(2):715-717.

10. Florkowski CM, Molyneux SL, George PM. (2008) Osuvastatin in older patients with systolic heart failure [J]. N Engl J Med.358 (12) : 1301.

11. Tavazzi L, Maggioni AP, et al. (2008) Effect of rosuvastatin in patients with chronic heart failure (the GISSI- HF trial):a randomised, double- blind, placebo-controlled trial [J]. Lancet. 372 (9645):1231-1239.

12. Ruilope LM, Dukat A, Bohm M, et al. (2010) Blood- pressure reduction with LCZ696, a novel dual- acting inhibitor of the angiotensin II receptor and neprilysin: a randomised, doubleblind, placebo- controlled, active comparator study [J]. Lancent, 375 (722): 1255-1266.

13. Mcmurray J, Packer M, Desai A, et al. (2014) A putative placebo analysis of the effects of LCZ696 on clinical outcomes in heart failure [J]. Eur Heart J. 36 (7) : 434-439.
14. Hsiao FC, Wang CL, Chang PC, Lu YY1, et al. (2020) Angiotensin Receptor Neprilysin Inhibitor for Patients With Heart Failure and Reduced Ejection Fraction: Real-World Experience From Taiwan. J Cardiovasc Pharmacol Ther. 25(2):152-157.

15. Grabowski M, Ozierański K, Balsam P, et al. (2019) The effect of sacubitril / valsartan on the occurrence of ventricular arrhythmia and the risk of sudden cardiac death in patients with chronic heart failure with reduced left ventricular ejection fraction. Kardiol Pol. 77(10):987-993.

16. Huo JY, Jiang WY, Chen C, et al. (2019) Effects of Angiotensin Receptor Neprilysin Inhibitors on Inducibility of Ventricular Arrhythmias in Rats with Ischemic Cardiomyopathy. Int Heart J. 27;60(5):1168-1175. .

17. Mebazaa A, Nieminen MS, Packer M, et al. (2007) Levosimendan vs dobutamine for patients with acute decompensated heart failure: the SURVIVE Randomized Trial [J]. JAMA, 297 ( 17) : 1883-1891.

18. Mcmurray J, Packer M, Desai A, et al. (2014) A putative placebo analysis of the effects of LCZ696 on clinical outcomes in heart failure [J]. Eur Heart J, 36 (7) : 434-439.

19. Scott D. Solomon MD, Adel R. et al.Efficacy and Safety of LCZ696 Compared to Valsartan, on Morbidity and Mortality in Heart Failure Patients With Preserved Ejection Fraction (PARAGON-HF).

20. Khariton Y, Fonarow GC, Arnold SV, et al. (2019) Association Between Sacubitril/Valsartan Initiation and Health Status Outcomes in Heart Failure With Reduced Ejection Fraction. JACC Heart Fail. 7(11):933-941.
This work is licensed under Creative Commons Attribution 4.0 License

To Submit Your Article Click Here: Submit Article

DOI: $10.31579 / 2693-4779 / 024$
Ready to submit your research? Choose Auctores and benefit from:

$$
\begin{aligned}
& \text { *ast, convenient online submission } \\
& \text { rigorous peer review by experienced research in your field } \\
& \text { rapid publication on acceptance } \\
& \text { * authors retain copyrights } \\
& \text { unique DOI for all articles } \\
& \text { immediate, unrestricted online access }
\end{aligned}
$$

At Auctores, research is always in progress.

Learn more www.auctoresonline.org/journals/clinical-research-andclinical-trials 